\title{
Exosomal connexin 43 regulates the resistance of glioma cells to temozolomide
}

\author{
ZHANG-JIAN YANG $^{1 *}$, LE-LING ZHANG ${ }^{1 *}$, QIU-CHEN BI ${ }^{1}$, LI-JUN GAN ${ }^{1}$, MIN-JUN WEI ${ }^{2}$, TAO HONG ${ }^{2}$, \\ REN-JIE TAN ${ }^{1}$, XUE-MEI LAN ${ }^{1}$, LI-HUA LIU ${ }^{1}$, XIAO-JIAN HAN ${ }^{3,4}$ and LI-PING JIANG ${ }^{1,3}$ \\ ${ }^{1}$ Department of Pharmacology, School of Pharmaceutical Science, Nanchang University; ${ }^{2}$ Department of Neurosurgery, \\ First Affiliated Hospital of Nanchang University; ${ }^{3}$ Key Laboratory of Drug Targets and Drug Screening of Jiangxi Province; \\ ${ }^{4}$ Department of Geriatrics, Jiangxi Provincial People's Hospital Affiliated to Nanchang University, \\ Nanchang, Jiangxi 330006, P.R. China
}

Received October 21, 2020; Accepted January 25, 2021

DOI: $10.3892 / o r .2021 .7995$

\begin{abstract}
Glioblastoma is the most common and aggressive brain tumor and it is characterized by a high mortality rate. Temozolomide (TMZ) is an effective chemotherapy drug for glioblastoma, but the resistance to TMZ has come to represent a major clinical problem, and its underlying mechanism has yet to be elucidated. In the present study, the role of exosomal connexin $43(\mathrm{Cx} 43)$ in the resistance of glioma cells to TMZ and cell migration was investigated. First, higher expression levels of Cx43 were detected in TMZ-resistant U251 (U251r) cells compared with those in TMZ-sensitive (U251s) cells. Exosomes from U251s or U251r cells (sExo and rExo, respectively) were isolated. It was found that the expression of $\mathrm{Cx} 43$ in rExo was notably higher compared with that in sExo, whereas treatment with rExo increased the expression of Cx43 in U251s cells. Additionally, exosomes stained with dioctadecyloxacarbocyanine (Dio) were used to visualized exosome uptake by glioma cells. It was observed that the uptake of Dio-stained rExo in U251s cells was more prominent compared with that of Dio-stained sExo, while ${ }^{37,43}$ Gap27, a gap junction mimetic peptide directed against $\mathrm{Cx} 43$, alleviated the rExo uptake by cells. Moreover, rExo increased the $\mathrm{IC}_{50}$
\end{abstract}

Correspondence to: Professor Li-Ping Jiang, Department of Pharmacology, School of Pharmaceutical Science, Nanchang University, 461 Bayi Avenue, Nanchang, Jiangxi 330006, P.R. China

E-mail: lipingjiang2002@aliyun.com

Professor Xiao-Jian Han, Key Laboratory of Drug Targets and Drug Screening of Jiangxi Province, 461 Bayi Avenue, Nanchang, Jiangxi 330006, P.R. China

E-mail: hanxiaojian@hotmail.com

*Contributed equally

Abbreviations: Cx43, connexin 43; TMZ, temozolomide; U251r, TMZ-resistant U251 cells; U251s, TMZ-sensitive U251 cells; Exo, exosome; Dio, dioctadecyloxacarbocyanine

Key words: glioma, temozolomide, drug resistance, exosomes, connexin 43 of U251s to TMZ, colony formation and Bcl-2 expression, but decreased Bax and cleaved caspase-3 expression in U251s cells. ${ }^{37,43}$ Gap27 efficiently inhibited these effects of rExo on U251s cells. Finally, the results of the wound healing and Transwell assays revealed that rExo significantly enhanced the migration of U251s cells, whereas ${ }^{37,43} \mathrm{Gap} 27$ significantly attenuated rExo-induced cell migration. Taken together, these results indicate the crucial role of exosomal $\mathrm{Cx} 43$ in chemotherapy resistance and migration of glioma cells, and suggest that $\mathrm{C} \times 43$ may hold promise as a therapeutic target for glioblastoma in the future.

\section{Introduction}

Glioma is a type of aggressive tumor originating from the neuroectoderm and accounts for $45-50 \%$ of all intracranial tumors (1). According to the 2007 WHO central nervous system tumor grading system, malignant gliomas, including glioblastoma, anaplastic astrocytoma, anaplastic oligodendrocyte tumor and anaplastic oligodendroglioma (WHO grade III-IV) account for more than half of all gliomas (2). Malignant glioma is a solid tumor composed of rapidly proliferating non-glial cells, and is characterized by intra- and intertumor heterogeneity, resistance to chemotherapy and poor prognosis (3). Due to its characteristic malignant invasive growth, the therapeutic approach has evolved from a single surgical approach to a combination of surgical approaches with radiotherapy and chemotherapy. However, the median survival of patients with glioblastoma only increased from 12.1 with radiotherapy alone to 14.6 months with radiotherapy plus temozolomide, and the prognosis of patients did not significantly improve (4).

Temozolomide (TMZ) is an oral DNA alkylating agent, which inactivates O6-methylguanine-DNA alkyl transferase (5), forms a notch for substrate DNA, and causes DNA double strand breaks and cell cycle arrest in the G2/M phase, eventually leading to cell apoptosis (6). TMZ readily crosses the blood-brain barrier; thus, it is one of the most effective chemotherapeutic drugs for glioma (7). However, $\mathrm{TMZ}$ is only $\sim 45 \%$ effective against malignant glioma (8), and a significant proportion of gliomas develop resistance to TMZ during treatment. The emergence of chemotherapy 
resistance and subsequent disease recurrence have become an urgent clinical problem in glioma (9). Most studies on TMZ resistance in gliomas are focused on drug efflux mediated by $\mathrm{ABC}$ transporters, repair of damaged DNA, changes in apoptotic pathways, cell cycle and cell metabolism $(10,11)$. All these mechanisms are closely intertwined, and may be implicated in the development of chemotherapy resistance (12). However, other underlying mechanisms may also be involved in the development of resistance. Thus, the exact molecular mechanisms underlying resistance of glioma to chemotherapy remain to be further elucidated.

Exosomes are lipid bilayer vesicles with a diameter of $30-150 \mathrm{~nm}$ that are actively produced by almost all cells $(13,14)$. Exosomes are developed from early endosomes, and usually express specific surface markers, including CD9, CD63, TSG101, Alix and Flotillin-1 (15). Exosomes contain non-coding RNAs, lipids and up to 7,000 proteins, and they mediate cell-cell communication by delivering these substances to surrounding cells (16). It was previously demonstrated that exosomes play an important role in the migration, invasion and chemotherapy resistance of cancer cells (17). Exosomes may alter the tumor microenvironment and promote drug resistance through transferring various drug resistance-related factors between drug-resistant and drug-sensitive cells, and between drug-resistant cells and tumor stromal cells (18-20). Most studies have focused on the role of exosomal contents, but a few also reported that exosomal membrane components may affect the recognition and uptake of exosomes by recipient cells (21). Exosomes interact with recipient cells through receptor-ligand interaction, endocytosis and membrane fusion (22). Phagocytic cells, such as macrophages, are more likely to endocytose exosomes (23). Non-phagocytic cells, such as T-lymphocyte subsets, express receptors for binding with the ligands on exosomes, thereby mediating membrane fusion and delivering exosomal contents to recipient cells (24). In the latter case, exosomal membrane components play an important role in the uptake of exosomes by recipient cells. Recently, it was reported that connexins (Cxs) may also be assembled into a hemi-channel in the membrane of exosomes secreted by cells (25). Therefore, it is crucial to investigate whether exosomal Cxs are involved in the interaction between exosomes and recipient cells.

Cxs are transmembrane proteins composed of two extracellular rings, an intracellular ring, an amino and a carboxyl tail domain (26). More than 20 Cxs have been identified in humans and mice (27). Cxs can oligomerize on the cell membrane to form gap junction hemi-channels, which are docked and assembled into gap junction channels to mediate gap junction intercellular communication. $\mathrm{Cx} 43$ is one of the most common Cxs, and is involved in tumor invasion, progression, and the resistance of glioma cells to TMZ (28-30). It has also been reported that the expression level of $\mathrm{Cx} 43$ in gliomas is higher compared with that in the adjacent tissues (31), which suggests the role of $\mathrm{Cx} 43$ in gliomagenesis. Moreover, upregulated expression of $\mathrm{Cx} 43$ through epidermal growth factor receptor-activated c-Jun N-terminal kinase/extracellular signal-regulated kinase 1/2/activator protein-1 signaling axis has been found in TMZ-resistant glioblastoma cell lines (32).

The present study was undertaken to investigate the role of exosomal Cx43 in the resistance of glioma cells to TMZ and cell migration. The expression of $\mathrm{Cx} 43$ was examined in TMZ-resistant and TMZ-sensitive U251 cells (U251r and $\mathrm{U} 251 \mathrm{~s}$, respectively) as well as in their exosomes (sExo and rExo, respectively). The effects of rExo on the $\mathrm{IC}_{50}$ of U251s cells to TMZ, colony formation ability, Bcl-2 expression, cell migration and Bax expression were also examined. Furthermore, it was investigated whether pretreatment with the gap junction mimetic peptide ${ }^{37,43}$ Gap27 would be able to inhibit the uptake of rExo by U251s cells, and alleviate rExo-induced TMZ resistance, colony formation and migration of U251s cells. The aim was to elucidate the role of exosomal Cx43 in chemotherapy resistance and migration of glioma cells, and determine whether it may serve as a therapeutic target for glioblastoma in the future.

\section{Materials and methods}

Chemicals and reagents. Fetal bovine serum (FBS) was purchased from EVERY GREEN (Biotech Co.). Dulbecco's modified Eagle's medium (DMEM), DMSO, PMSF, crystal violet, $4 \%$ paraformaldehyde, anti-fluorescence attenuation sealant, trypsin and penicillin-streptomycin solution (P/S) were purchased from Beijing Solarbio Science \& Technology Co., Ltd. Tween-20 and TEMED were purchased from Sigma-Aldrich; Merck KGaA. TMZ was obtained from MedChemExpress. ${ }^{37,34} \mathrm{Gap} 27$ was purchased from Eurogentec. PrimeScript ${ }^{\mathrm{TM}}$ RT reagent Kit (cat. no. RR037A) with gDNA Eraser and QuantiNova ${ }^{\mathrm{TM}} \mathrm{SYBR}^{\circledR}$-Green PCR Kit (cat. no. RR420A) were obtained from Takara Bio, Inc.

Cell culture. U251s and U251r cells were purchased from the American Type Culture Collection. The human U251s and U251r cell lines were grown in DMEM supplemented with $10 \% \mathrm{FBS}$ and $100 \mu \mathrm{g} / \mathrm{ml} \mathrm{P} / \mathrm{S}$ in a humidified incubator at $37^{\circ} \mathrm{C}$ with an atmosphere containing $5 \% \mathrm{CO}_{2}$.

Reverse transcription-quantitative PCR (RT-PCR). Total RNA was isolated from cells using Eastep ${ }^{\circledR}$ Super (Promega Corporation). Reverse transcription was performed using PrimeScript ${ }^{\mathrm{TM}} \mathrm{RT}$ reagent Kit (Takara Bio, Inc.) at $37^{\circ} \mathrm{C}$ for $15 \mathrm{~min}, 85^{\circ} \mathrm{C}$ for $5 \mathrm{sec}$ and then held at $4^{\circ} \mathrm{C}$. qPCR was performed using TB Green ${ }^{\circledR}$ Premix Ex Tap ${ }^{\mathrm{TM}}$ (Takara Bio, Inc.) on CFX Connect ${ }^{\mathrm{TM}}$ Real-time System (Bio-Rad Laboratories, Inc.) at $95^{\circ} \mathrm{C}$ for $30 \mathrm{sec}$, for 39 thermal cycles $\left(95^{\circ} \mathrm{C}\right.$ for $5 \mathrm{sec}$ and $60^{\circ} \mathrm{C}$ for $30 \mathrm{sec}$ ), with GAPDH serving as the internal control. The sequences of the primers used for RT-PCR are presented in Table I.

Western blotting. Cells were harvested and lysed by RIPA lysis buffer (Beijing Solarbio Science \& Technology Co., Ltd.) according to the manufacturer's instructions. Protein concentrations were determined with BCA Protein Assay Kit (Applygen Technologies, Inc.). Protein samples (18 $\mu \mathrm{g})$ were separated by $10 \%$ SDS-PAGE (Bio-Rad Laboratories, Inc.) and transferred to PVDF membranes by Trans-Blot SD type membrane transfer system (Bio-Rad Laboratories, Inc.). After blocking with 5\% skimmed milk for $1 \mathrm{~h}$ at room temperature, proteins on the PVDF membranes were immunoblotted for $2 \mathrm{~h}$ at room temperature using primary antibodies for $\mathrm{Cx} 43$ (cat. no. ab79010; Abcam, 1:1,000), Bax (cat. no. 2772; Cell 
Table I. Primer sequences.

Sequences $\left(5^{\prime} \rightarrow 3^{\prime}\right)$

\begin{tabular}{llc}
\cline { 2 - 3 } Gene names & \multicolumn{1}{c}{ Forward } & \multicolumn{1}{c}{ Reverse } \\
\hline Connexin 43 & CAAAATCGAATGGGGAGGC & GCTGGTCCACAATGGCTAGT \\
GAPDH & GTCAAGGCTGAGAACGGGAA & AAATGAGCCCCAGCCTTCTC
\end{tabular}

Signaling Technology, Inc., 1:1,000), Bcl-2 (cat. no. YT0407; ImmunoWay, 1:1,000), cleaved caspase-3 (cat. no. 9664; Cell Signaling Technology, Inc., 1:1,000), TSG101 and CD63 (cat. no. DF8427 and cat. no. AF5117; Affinity Biosciences, 1:1,000) and GADPH (cat. no. 70004; Affinity Biosciences, 1:2,000). After washing for 3 times, the membranes were further incubated with HRP-conjugated goat anti-mouse (cat. no. GAM007; MultiSciences Biotech Co., Ltd., 1:2,000) or goat anti-rabbit (cat. no. BA1060; Boster Biological Technology Co., Ltd., 1:2,000) antibodies at room temperature for $1 \mathrm{~h}$. The chemiluminescence signals were detected with an enhanced chemiluminescence assay kit (Beijing Kangwei Century Biotechnology Co., Ltd.). Densitometric analysis was conducted with the Gel Imaging System (Analytik Jena US LLC).

Isolation and identification of exosomes. Exosomes were isolated by differential velocity centrifugation. In brief, culture medium was initially subjected to centrifugation at $300 \mathrm{x} \mathrm{g}$ for $10 \mathrm{~min}, 2,000 \mathrm{x} \mathrm{g}$ for $10 \mathrm{~min}$ and $10,000 \mathrm{x} \mathrm{g}$ for $30 \mathrm{~min}$ at $4^{\circ} \mathrm{C}$. The supernatant was further subjected to centrifugation at $100,000 \mathrm{x} \mathrm{g}$ for $70 \mathrm{~min}$ at $4^{\circ} \mathrm{C}$, and pellets rich in exosomes were resuspended in $1 \mathrm{X}$ PBS. To identify the isolated exosomes, the particle size of exosomes was initially determined by Zetasizer Nano ZS 90 particle size analyzer (Malvern Panalytical), and the morphology of the exosomes was observed under a transmission electron microscope (Leica Microsystems $\mathrm{GmbH}$; magnification, x40,000). As described previously (33), $10 \mu \mathrm{l}$ of sample was added to a 2-mm copper mesh grid and the excess PBS was removed with a filter paper. Then, $10 \mu \mathrm{l}$ of phosphotungstate $(20 \mathrm{~g} / \mathrm{l})$ was added to the copper net for negative staining for $2 \mathrm{~min}$ and grilled under an incandescent lamp for $5 \mathrm{~min}$. The isolated samples were subjected to $10 \%$ SDS-PAGE, and western blotting was used to detect the marker proteins TSG101 and CD63 on exosomes.

Exosome uptake experiment. To label exosomes, $10 \mu \mathrm{M}$ of dioctadecyloxacarbocyanine (Dio) membrane dye was added to the exosomal suspension in a $1: 1$ ratio and incubated at $37^{\circ} \mathrm{C}$ in the dark for $30 \mathrm{~min}$, followed by washing with PBS and centrifugation at $100,000 \mathrm{x}$ g for $70 \mathrm{~min}$ for 3 times to remove the free Dio dye. The Dio-labeled exosomes were resuspended with sterile $1 \mathrm{X}$ PBS. The exosome concentration was detected by the BCA Protein Assay. Three concentrations of 25, 50 and $100 \mu \mathrm{g} / \mathrm{ml}$ were used in the cell treatment experiments. The results revealed that the effect of rExo at $25 \mu \mathrm{g} / \mathrm{ml}$ was weak, while rExo at 50 and $100 \mu \mathrm{g} / \mathrm{ml}$ markedly affected Cx43 expression in U251 cells. Therefore, the concentration of exosomes at $50 \mu \mathrm{g} / \mathrm{ml}$ was considered sufficient and was used for the following cell treatment experiments. In the control groups, the same volume of PBS was added to the cells. To detect uptake of exosomes, U251s cells were incubated with $50 \mu \mathrm{g} / \mathrm{ml}$ of Dio-labeled rExo or sExo for $30 \mathrm{~min}$ at $37^{\circ} \mathrm{C}$ in the dark. Then, the cells were washed twice with PBS, and fixed with $4 \%$ paraformaldehyde for $10 \mathrm{~min}$ on ice. The nuclei of U251s cells were counterstained with $500 \mu \mathrm{l}$ of Hoechst $33342(10 \mu \mathrm{g} / \mathrm{ml})$ for $5 \mathrm{~min}$ at room temperature in the dark. Finally, the cells were washed three times with PBS. The uptake of Dio-labeled exosomes by cells was observed under an inverted fluorescence microscope (Leica Microsystems $\mathrm{GmbH}$; magnification, $\mathrm{x} 400$ ).

Cell viability detection. The Cell Counting Kit-8 (CCK-8; Vazyme Biotech Co., Ltd.) assay was performed to evaluate the viability of U251s and U251r cells according to the manufacturer's instructions. Briefly, $\sim 4 \times 10^{3}$ cells were seeded on 96-well plates and cultured in full growth medium. After incubation with TMZ at 0, 100, 200, 400, 600, 800, 1,000 and $1,200 \mu \mathrm{M}$ for $72 \mathrm{~h}, 10 \mu \mathrm{l}$ of CCK-8 was added to each well, and cells were further incubated with CCK- 8 for $\sim 2 \mathrm{~h}$. The absorbance at $450 \mathrm{~nm}$ was measured spectrophotometrically using a microplate reader (Bio-Rad Laboratories, Inc.). Control and blank wells were also detected. All assays were performed at least three times.

Colony formation assay. U251s Cells were incubated with $1 \mathrm{X}$ PBS, $50 \mu \mathrm{g} / \mathrm{ml}$ of sExo or rExo and rExo $+200 \mu \mathrm{M}^{37,43} \mathrm{Gap} 27$ for $48 \mathrm{~h}$. After incubation, cells were harvested and resuspended to a density of $6 \times 10^{3} / \mathrm{ml}$. a total of $50 \mu \mathrm{l}$ cell suspension was inoculated in a 6-well plate, and was further cultured for 10 days. Then, the cells were washed with $1 \mathrm{X}$ PBS and fixed in $4 \%$ paraformaldehyde for $15 \mathrm{~min}$ on ice. A total of $2 \mathrm{ml}$ of crystal violet solution $(0.1 \%)$ was added to each well and the cells were stained for $30 \mathrm{~min}$ at room temperature. After washing 3 times with $1 \mathrm{X} \mathrm{PBS}$, the number of colonies with $>50$ cells was counted.

Wound healing and Transwell assays. The wound healing and Transwell assays were performed as previously described (34). For the wound healing assay, $3 \times 10^{5} \mathrm{U} 251$ s cells were seeded on $35-\mathrm{mm}$ culture dishes. When the cell confluence reached $\sim 90 \%$, a scratch was made in monolayer of cells with a $200-\mu 1$ pipette tip. After removing unattached cells, U251 cells were incubated with 1X PBS, $50 \mu \mathrm{g} / \mathrm{ml}$ of sExo or rExo and $\mathrm{rExo}$ $+200 \mu \mathrm{M}^{37,43} \mathrm{Gap} 27$ for $48 \mathrm{~h}$. At 0,24 and $48 \mathrm{~h}$ of incubation with the aforementioned reagents, the cells were photographed for evaluation of wound closure under an inverted microscope (DMi8; Leica Microsystems GmbH; magnification, x100). ImageJ software (1.52v; National Institutes of Health) was used to calculate the area of migration. Transwell chambers 
A

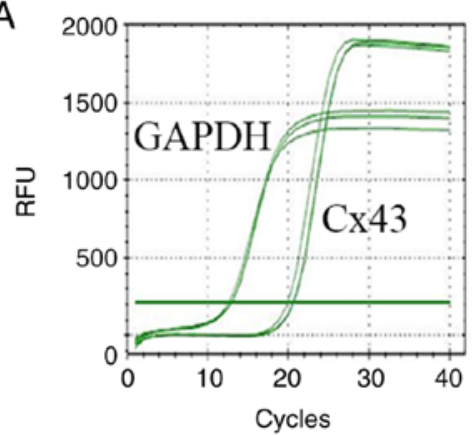

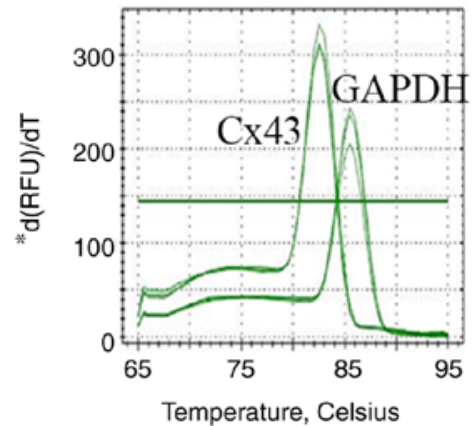

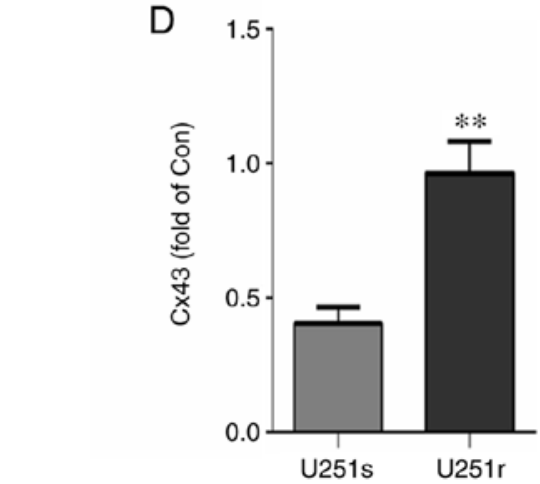

B

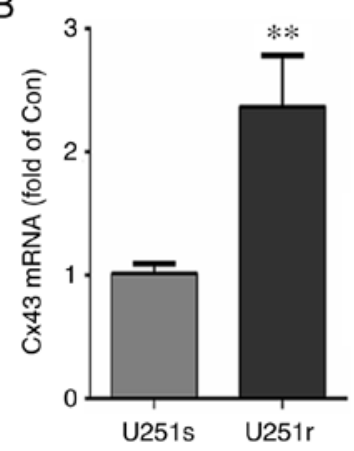

C

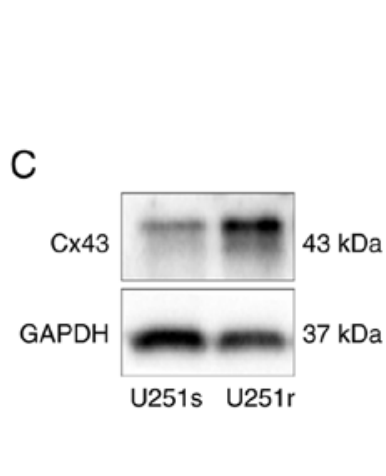

Figure 1. Cx43 mRNA and protein levels in U251s and U251r cells. (A and B) Cx43 mRNA levels in U251s and U251r cells were detected by reverse transcription-quantitative PCR. The transcription of GAPDH was used as endogenous control. (C and D) Cx43 protein expression level in U251s and U251r cells. GAPDH was used as endogenous control. (B and D) Cx43 mRNA and protein levels in U251s and U251r cells were quantitatively analyzed from data of at least three independent experiments. ${ }^{* *} \mathrm{P}<0.01$. Cx43, connexin 43; U251r, temozolomide-resistant U251 glioma cells; U251s, temozolomide-sensitive U251 glioma cells; RFU, relative fluorescence units.

(Corning, Inc.) were used for Transwell assay. Briefly, $3 \times 10^{6}$ U251s cells incubated with $1 \mathrm{X}$ PBS, $50 \mu \mathrm{g} / \mathrm{ml}$ of sExo or rExo and rExo $+200 \mu \mathrm{M}^{37,43} \mathrm{Gap} 27$ for $48 \mathrm{~h}$ were inoculated in the upper chamber with $200 \mu \mathrm{l}$ serum-free medium. The upper chamber was then incubated in a 24-well plate chamber with complete medium containing $10 \%$ FBS for $24 \mathrm{~h}$. After removing the cells on the top surface of the upper chamber, the migrated cells on lower surface of the chamber were fixed in $4 \%$ paraformaldehyde solution for $10 \mathrm{~min}$ at room temperature, then stained in $0.1 \%$ crystal violet solution for $30 \mathrm{~min}$ at room temperature. The migrated cells were photographed under an inverted microscope (DMi8; Leica Microsystems GmbH) and counted in four random fields at a magnification of $\mathrm{x} 200$.

Statistical analysis. The data in the present study were analyzed with GraphPad Prism v.5 (GraphPad Software, Inc.) and are presented as the mean $\pm \mathrm{SD}$. The statistical significance of the differences among multiple groups was analyzed by one-way ANOVA parametric testing followed by Tukey's multiple comparisons test. In the case of comparison between two groups, unpaired two-tailed Student's t-test was performed. $\mathrm{P}<0.05$ was considered to indicate statistically significant differences.

\section{Results}

Cx43 expression is upregulated in TMZ-resistant U251r cells. To investigate the role of $\mathrm{Cx} 43$ in the chemotherapy resistance of glioblastoma cells, the mRNA and protein levels of Cx43 were measured in U251r and U251s cells using RT-PCR and western blotting assays, respectively. As shown in Fig. 1A and B, the mRNA level of Cx43 in U251r cells was significantly higher compared with that in U251s cells. Similarly, the results of western blotting demonstrated that the $\mathrm{Cx} 43$ protein level was also significantly higher in U251r cells compared with that in U251s cells (Fig. 1C and D; $\mathrm{P}<0.01)$. These results are consistent with those of previous studies $(31,32)$, and suggest that $\mathrm{Cx} 43$ may be involved in the resistance of glioblastoma cells to TMZ.

Isolation and identification of exosomes from U251s and U251r cells. First, exosomes were isolated from U251s and U251r cells by differential velocity centrifugation, as previously described (35). After isolation, the morphology of the vesicles was observed under a transmission electron microscope (Fig. 2A). It has been reported that exosomes are lipid bilayer vesicles with a diameter of 30-150 nm. The samples isolated from U251s and U251r cells were subjected to Zetasizer Nano ZS 90 particle size analyzer. As shown in Fig. 2B, the size distribution of the vesicles was concentrated in the range of $40-120 \mathrm{~nm}$. Thus, the morphology and particle size distribution of vesicles isolated from cells were found to be consistent with the characteristics of exosomes. Furthermore, western blotting was used to detect the expression of exosomal markers, including CD63 and TSG101, in isolated samples. The results revealed that a strong signal of CD63 and TSG101 was detected in samples isolated from U251s and U251r cells, while the signal was notably weaker in whole-cell lysate from U251s cells (Fig. 2C-E). These results indicate that the exosomes (sExo and $\mathrm{rExo}$ ) were successfully isolated from U251s and U251r cells, respectively. 
A
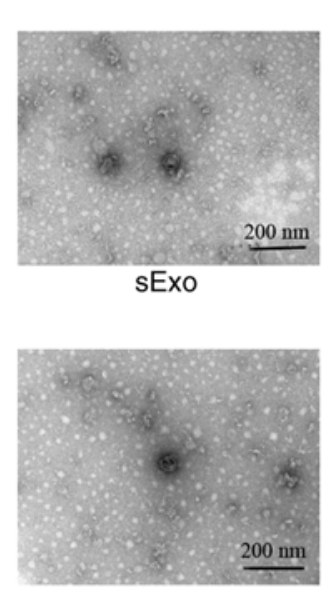

rExo
B
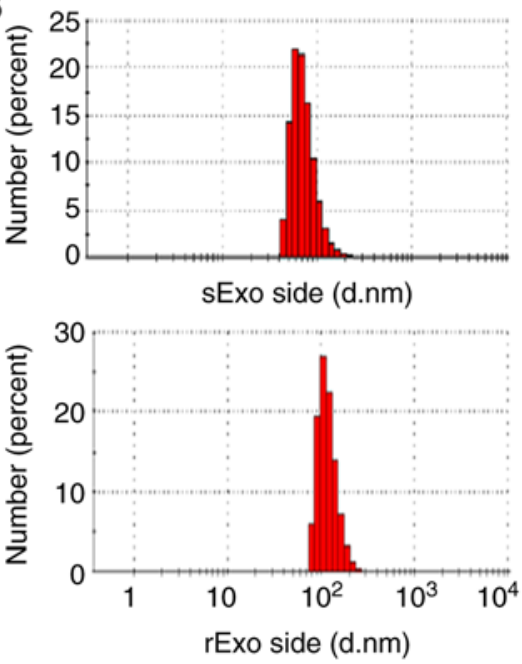

D

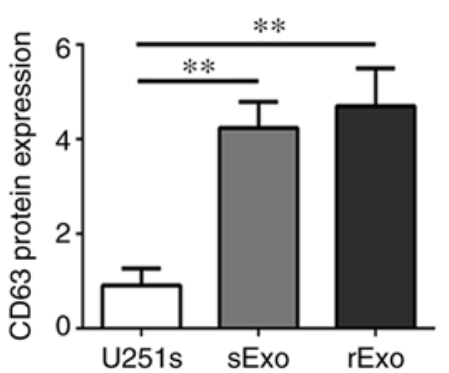

C

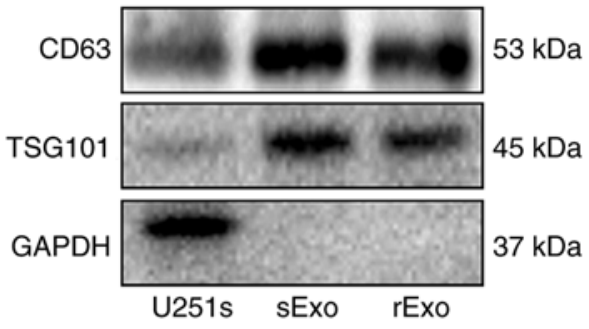

Figure 2. Isolation and identification of exosomes from U251s and U251r cells. (A) Exosomes were isolated from U251s or U251r cells by differential velocity centrifugation, and representative images of sExo and rExo were captured under a transmission electron microscope. Scale bar, $200 \mathrm{~nm}$. (B) sExo and rExo diameters were measured using the Zetasizer Nano ZS90 particle size analyzer. The size distribution of the vesicles was concentrated in the range of 40-120 nm. (C) Western blotting was used to examine the expression of exosomal markers CD63 and TSG101 in U251s cells, sExo and rExo; GAPDH was used as an endogenous reference. ( $\mathrm{D}$ and E) Quantitative analysis of the protein expression level of CD63 and TSG101. ${ }^{* * *} \mathrm{P}<0.01,{ }^{* * *} \mathrm{P}<0.001$. U251r, temozolomide-resistant U251 glioma cells; U251s, temozolomide-sensitive U251 glioma cells; sExo, exosomes of U251s cells; rExo, exosomes of U251r cells.

rExo upregulates CX43 expression in U251s cells and facilitates exosome uptake via $C x 43$. It has been reported that Cxs can be assembled into a hemi-channel in the exosomal membrane (24). To explore the role of Cx43 in TMZ resistance, the expression of $\mathrm{Cx} 43$ in sExo and rExo was detected by western blotting. As shown in Fig. 3A and C, the expression of $\mathrm{Cx} 43$ in rExo was significantly higher compared with that in sExo $(\mathrm{P}<0.005)$. In addition, the effect of sExo and rExo on Cx43 expression in glioma cells was examined. U251s cells were incubated with $50 \mu \mathrm{g} / \mathrm{ml} \mathrm{sExo}, \mathrm{rExo}$ and PBS for $48 \mathrm{~h}$. Of note, the expression of Cx43 in U251s cells incubated with rExo was significantly increased, while there was no significant change in the expression of $\mathrm{Cx} 43$ in U251s cells treated with PBS or sExo (Fig. 3B and D). Next, the role of Cx43 in exosome uptake by glioma cells was further explored. U251s cells were incubated with Dio-sExo, Dio-rExo or Dio-rExo $+{ }^{37,43}$ Gap27. As shown in Fig. 3E, the uptake of sExo and rExo by U251s cells was observed. Consistent with exosomal Cx43 expression level, the fluorescence intensity of Dio-rExo in U251s cells was found to be stronger compared with that of Dio-sExo groups. Moreover, the gap junction mimetic peptide ${ }^{37,43} \mathrm{Gap} 27$ directed against exosomal Cx43 markedly alleviated the fluorescence intensity of Dio-rExo in U251s cells. These results suggest that exosomal Cx43 not only upregulates Cx43 expression in U251s cells, but also facilitates exosome uptake via Cx43.
Exosomal CX43 regulates TMZ resistance and colony formation ability of U251s cells. The aforementioned experiments demonstrated that exosomal $\mathrm{Cx} 43$ facilitated exosome uptake by U251s cells. Next, the role of exosomal Cx43 in development of TMZ resistance was examined. U251s cells were separately pretreated with PBS, sExo, rExo or rExo $+{ }^{37,43} \mathrm{Gap} 27$, followed by treatment with $0-800 \mu \mathrm{M}$ TMZ. The results of the CCK-8 assay demonstrated that the $\mathrm{IC}_{50}$ value of $\mathrm{U} 251$ s cells to $\mathrm{TMZ}$ was $\sim 220 \mu \mathrm{M}$ in the PBS and sExo groups, while the $\mathrm{IC}_{50}$ value of U251s cells to TMZ was increased to $580 \mu \mathrm{M}$ in the rExo group (Fig. 4A and B). Additionally, ${ }^{37,43} \mathrm{Gap} 27$ significantly alleviated the rExo-induced increase in the $\mathrm{IC}_{50}$ value of U251s cells to TMZ $(\mathrm{P}<0.001)$. Furthermore, the effect of exosomal Cx43 on the colony formation ability of U251s cells was examined. As shown in Fig. $4 \mathrm{C}$ and $\mathrm{D}$, the colony number in the PBS and sExo groups was $25 \pm 6$ and $38 \pm 4$, respectively. By contrast, rExo significantly increased the colony number to $76 \pm 6(\mathrm{P}<0.001)$, whereas treatment with $\mathrm{rExo}+{ }^{37,43} \mathrm{Gap} 27$ decreased the colony number to $51 \pm 7(\mathrm{P}<0.01)$. These results suggest that exosomal $\mathrm{Cx} 43$ enhances TMZ resistance and colony formation ability in glioma cells.

Exosomal Cx43 regulates the expression of Bcl-2, Bax and cleaved caspase-3 in U251s cells. The antineoplastic effect of TMZ is mediated by inducing intrinsic apoptosis and cell cycle arrest. Bcl-2 family proteins and caspases are important 
A
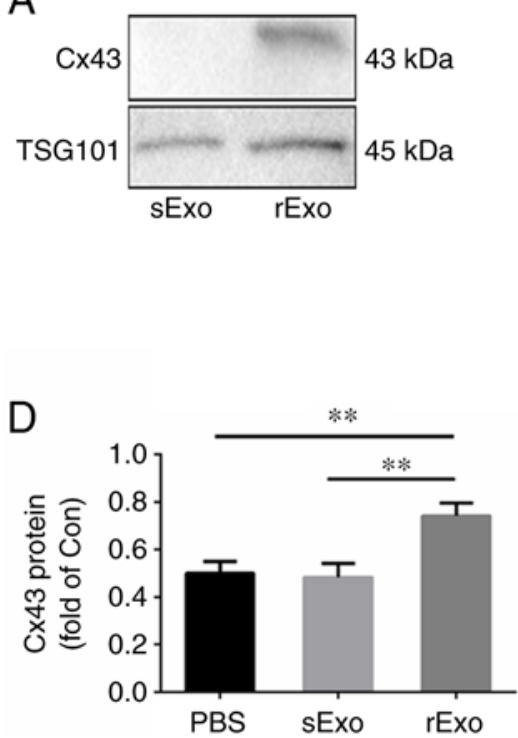

B

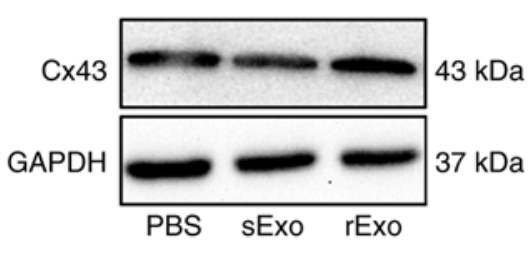

$E$

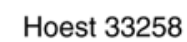

Hoest 33258

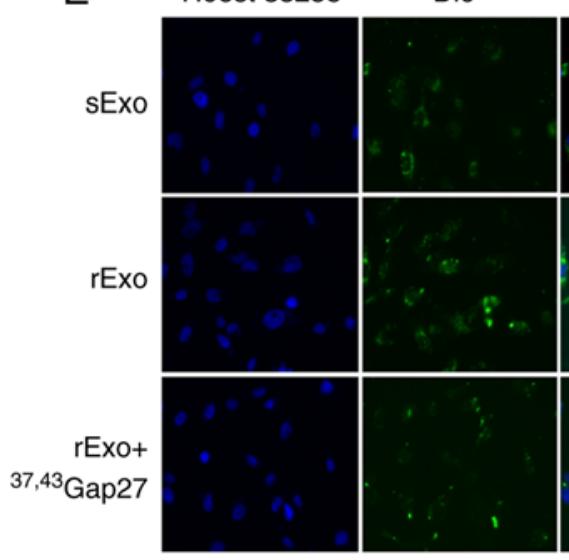

C

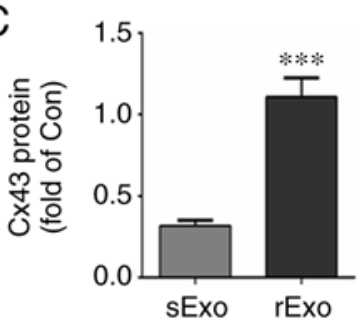

Merge

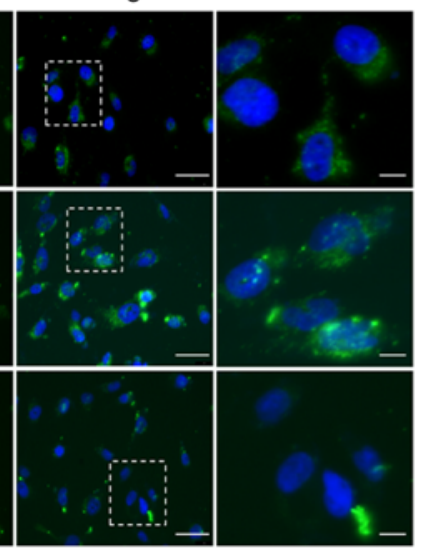

Figure 3. Role of exosomal Cx43 in exosome uptake by glioma cells. (A) Expression level of Cx43 in sExo and rExo. GAPDH was used as endogenous reference. (B) Expression of $\mathrm{Cx} 43$ in U251s cells after treatment with PBS, sExo and rExo for $48 \mathrm{~h}$. GAPDH was used as endogenous reference. (C and D) The relative expression level of $\mathrm{Cx} 43$ was indicated as the ratio of $\mathrm{Cx} 43 / \mathrm{GAPDH}$ in each group. Data represent the results of at least three independent experiments for each condition. ${ }^{* *} \mathrm{P}<0.01,{ }^{* * *} \mathrm{P}<0.001$. (E) Exosome uptake in U251s cells after treatment with Dio-sExo, Dio-rExo and Dio-rExo $+{ }^{37,43}$ Gap27. U251s cells were incubated with Dio-stained sExo, rExo and Dio-rExo $+{ }^{37,43}$ Gap27 for 30 min, then were counterstained with Hoechst 33342 (blue) for 5 min. Dio-stained exosome uptake (green) in cells was observed under fluorescence microscope. Scale bar, $100 \mu \mathrm{m}$. Cx43, connexin 43; U251r, temozolomide-resistant U251 glioma cells; U251s, temozolomide-sensitive U251 glioma cells; sExo, exosomes of U251s cells; rExo, exosomes of U251r cells; Dio, dioctadecyloxacarbocyanine.
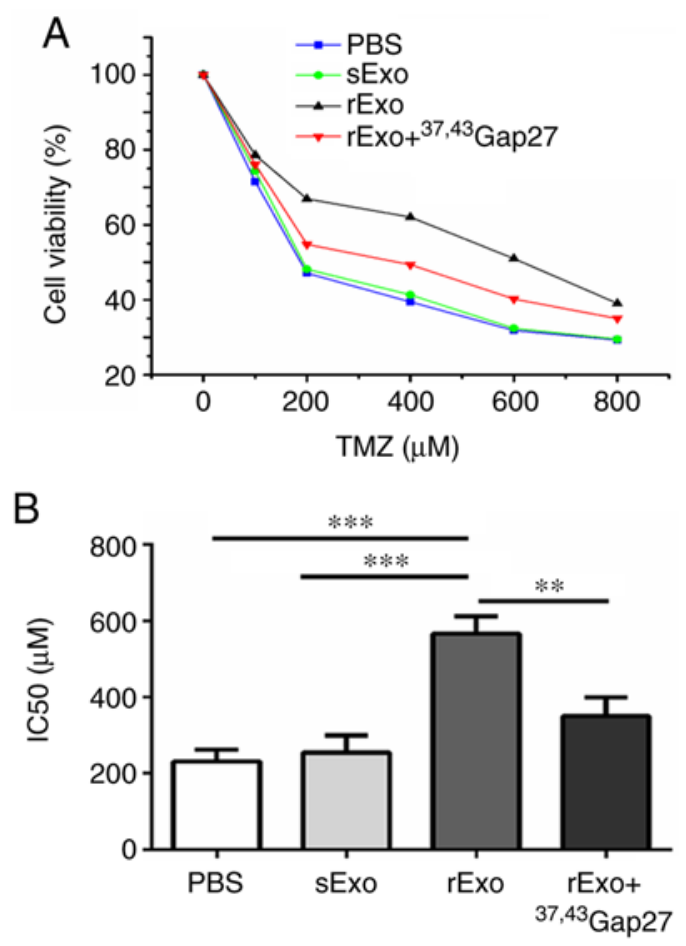

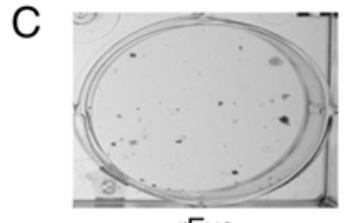

rExo

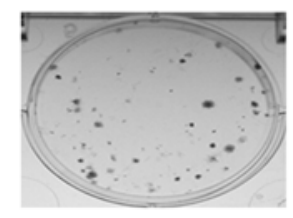

PBS
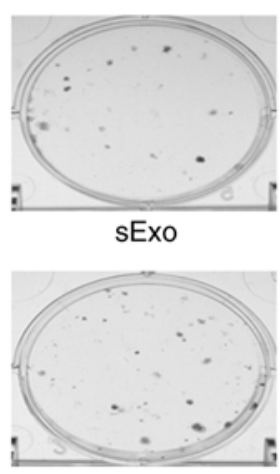

rExo+ ${ }^{37,43}$ Gap27

D

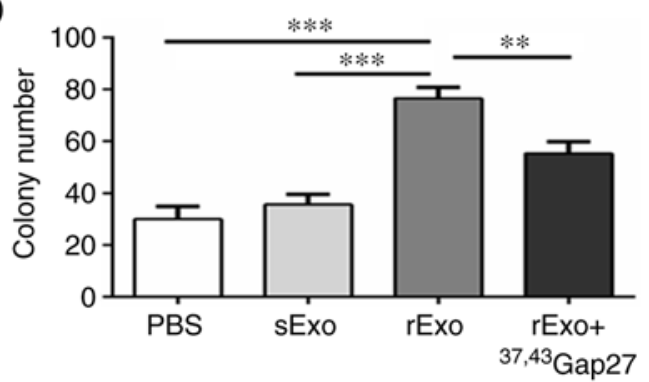

Figure 4. Effect of exosomal Cx43 on TMZ resistance and colony formation ability of U251s cells. (A) U251s cell viability in each group was detected using Cell Counting Kit-8 assay at $72 \mathrm{~h}$ after treatment with TMZ, and the cell viability at each TMZ concentration was plotted for the dose-response curve. (B) $\mathrm{IC}_{50}$ values of U251s cells to TMZ in the PBS, sExo, rExo and rExo $+{ }^{37,43} \mathrm{Gap} 27$ groups. Data represent the mean of at least three independent experiments for each condition. ${ }^{* *} \mathrm{P}<0.01,{ }^{* * * *} \mathrm{P}<0.001$. (C) Colony formation assay was conducted in U251s cells treated with PBS, sExo, rExo and rExo $+{ }^{37,43}$ Gap27. The representative images showed the colony morphology in each group. (D) Quantification of the number of colonies for statistical analysis. Data represent the results of three independent experiments for each group. ${ }^{* * *} \mathrm{P}<0.01,{ }^{* * *} \mathrm{P}<0.001$. Cx43, connexin 43; TMZ, temozolomide; U251r, TMZ-resistant U251 glioma cells; U251s, TMZ-sensitive U251 glioma cells; sExo, exosomes of U251s cells; rExo, exosomes of U251r cells. 
A

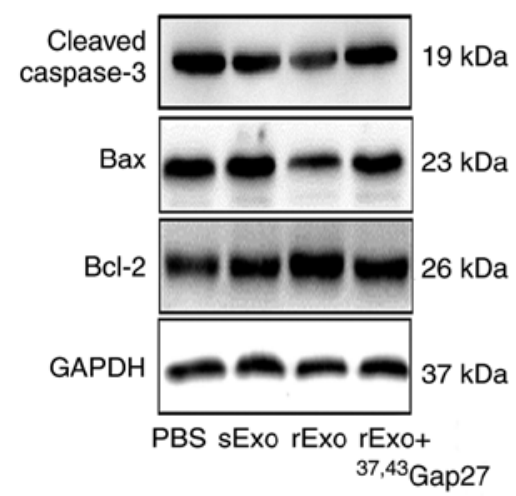

B

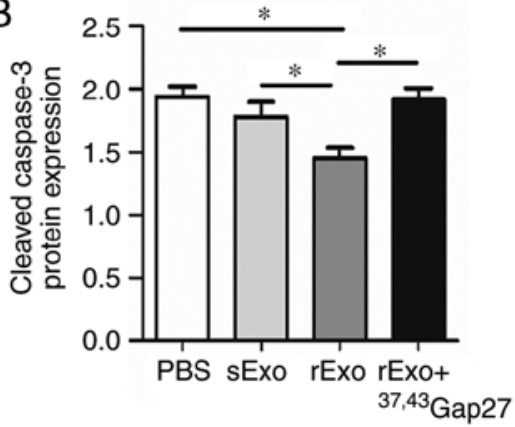

D

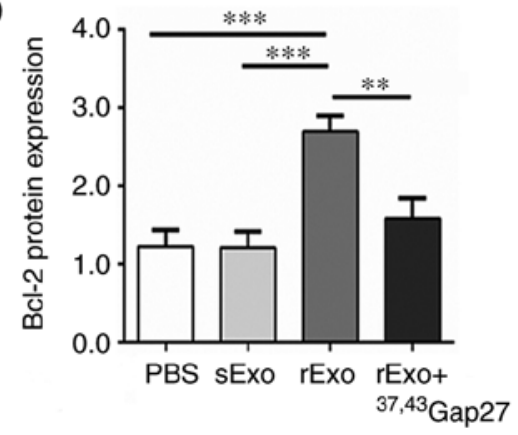

Figure 5. Effect of exosomal Cx43 on the expression of Bcl-2, Bax and cleaved caspase-3 in U251s cells. (A) Western blotting was used to detect the expression level of Bax, Bcl-2 and cleaved caspase-3 in U251s treated with PBS, sExo, rExo and rExo $+{ }^{37,43}$ Gap27. GAPDH was used as endogenous control. (B, C and D) Relative expression levels of Bax, Bcl-2 and cleaved caspase-3 were indicated as the Bax/GAPDH, Bcl-2/GAPDH and cleaved caspase-3/GAPDH ratios in each group. Data represent the results of at least three independent experiments for each condition. ${ }^{*} \mathrm{P}<0.05,{ }^{* *} \mathrm{P}<0.01,{ }^{* * *} \mathrm{P}<0.001$. Cx43, connexin 43 ; $\mathrm{U} 251 \mathrm{r}$, temozolomide-resistant U251 glioma cells; U251s, temozolomide-sensitive U251 glioma cells; sExo, exosomes of U251s cells; rExo, exosomes of U251r cells.

regulators of the intrinsic apoptosis pathway, which includes the pro-apoptotic (Bax), anti-apoptotic (Bcl-2) members and the cleavage of caspase-3. It has been reported that TMZ increases the Bax/Bcl-2 ratio in glioma cells, and dexamethasone induces $\mathrm{TMZ}$ resistance by maintaining the $\mathrm{Bax} / \mathrm{Bcl}-2$ ratio (36). Thus, effect of rExo on the expression of $\mathrm{Bcl}-2$, Bax and cleaved caspase-3 in U251s cells was examined. Compared with the PBS and sExo groups, the expression of Bax and cleaved caspase- 3 was significantly decreased and that of Bcl-2 was significantly increased in the rExo group. ${ }^{37,43}$ Gap27 efficiently attenuated the rExo-induced changes in the expression of Bax, Bcl-2 and cleaved caspase-3 in U251s cells (Fig. 5A-C). These results indicated that exosomal Cx43 may enhance TMZ resistance through modulating the expression of pro-apoptotic and anti-apoptotic proteins and activation of caspase signaling.

Exosomal CX43 enhances the migration ability of U251s cells. Glioma is a highly aggressive brain tumor. The strong migratory and invasive ability of glioma cells are closely associated with the poor prognosis of glioma. The present study further examined the effect of exosomal Cx43 on the migration ability of U251s cells. In the wound healing experiments, the migration of U251s cells at 0,24 and $48 \mathrm{~h}$ in each group was photographed. As shown in Fig. 6A and B, rExo significantly increased the wound closure at 24 and $48 \mathrm{~h}$ compared to the PBS and sExo groups. ${ }^{37,43}$ Gap27 efficiently inhibited rExo-induced wound closure in U251s cells. Consistently, the results of the Transwell assay also demonstrated that rExo enhanced the migration of U251s cells, whereas ${ }^{37,43} \mathrm{Gap} 27$ significantly alleviated rExo-induced cell migration (Fig. 6C and D). The results of the wound healing and Transwell assays suggest that $\mathrm{Cx} 43$ in rExo is involved in the regulation of glioma cell migration.

\section{Discussion}

Glioma is one of the most common primary malignant brain tumors. Although TMZ is widely used for the treatment of primary and metastatic brain cancer, a considerable proportion of gliomas are refractory to TMZ or gradually develop resistance, which is the main reason for the failure of chemotherapy for glioma (37). It has been demonstrated that exosomes from glioma cells can promote tumor progression by transferring oncogenic and immunomodulatory proteins and nucleic acids between different cancer cell subsets or between cancer cells and surrounding stromal cells (38), which plays an important role in chemotherapy resistance. On the other hand, treatment with neuropilin-1-targeted peptide (RGE)-modified, superparamagnetic iron oxide nanoparticle (SPION)- and curcumin (Cur)-loaded exosomes, named RGE-Exo-SPION/Cur, has been reported to target glioma and prolong the survival time of tumor-bearing mice (39). Thus, it is necessary to further investigate the mechanisms underlying the role of exosomes in the resistance of glioma cells to TMZ.

It has been reported that $\mathrm{Cxs}$ are assembled into a hemi-channel in the exosomal membrane (24). The hemi-channel formed by Cxs on the plasma membrane can promote communication between the cytoplasm and the extracellular environment (40). Cx43 is the most common gap junction protein and is highly expressed in primary glioma cell lines (41). It has been reported that high expression of $\mathrm{Cx} 43$ is 

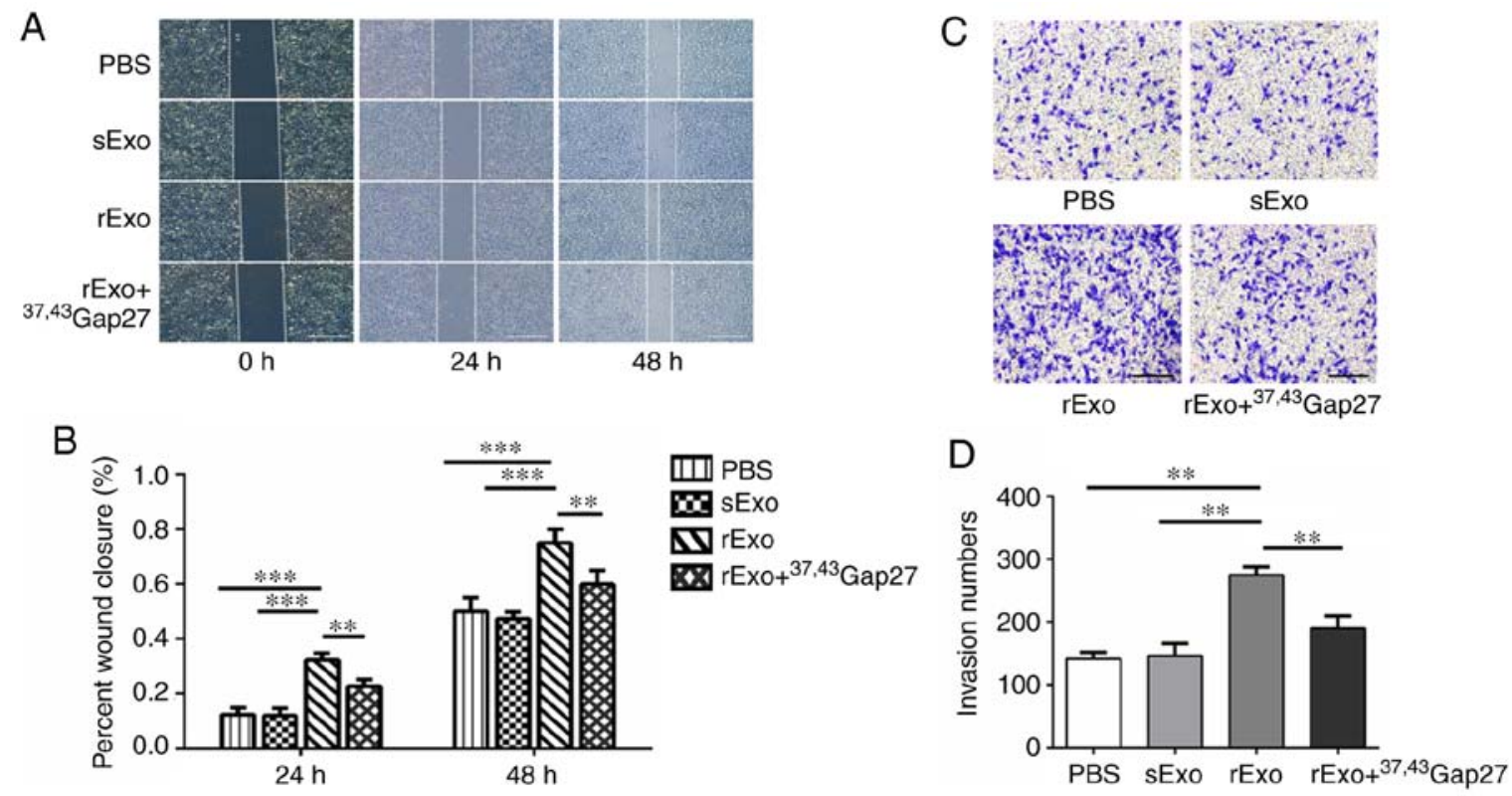

Figure 6. Exosomal Cx43 enhances the migration ability of U251s cells. (A) Wound healing experiments were performed to evaluate the migration of U251s cells at 0,24 and $48 \mathrm{~h}$ after treatment with PBS, sExo, rExo and $\mathrm{rExo}+{ }^{37,43} \mathrm{Gap} 27$. Scale bar, $200 \mu \mathrm{m}$. (B) Wound closure rate was measured using ImageJ software for statistical analysis. Data represent the results of at least three independent experiments for each group. ${ }^{* * *} \mathrm{P}<0.01,{ }^{* * * *} \mathrm{P}<0.001$. (C) Transwell assay was used to examine the migration of U251s cells after treatment with PBS, sExo, rExo and rExo $+{ }^{37,43} \mathrm{Gap} 27$. Scale bar, $200 \mu \mathrm{m}$. (D) The migrated U251s cells were counted in at least 9 fields from three independent experiments for each treatment. ${ }^{* *} \mathrm{P}<0.01$. Cx43, connexin 43; U251r, temozolomide-resistant $\mathrm{U} 251$ glioma cells; U251s, temozolomide-sensitive U251 glioma cells; sExo, exosomes of U251s cells; rExo, exosomes of U251r cells.

associated with the development of resistance of glioma cells to TMZ (31). In addition, a Cx43 C-terminus (CT) mimetic peptide-dubbed $\alpha \mathrm{CT} 1$ (a selective inhibitor of $\mathrm{Cx} 43$ channels) combined with TMZ significantly blocked the growth of human LN229/GSC tumors in mice (41). Consistently, in the present study, higher expression of Cx43 was detected in $\mathrm{U} 251 \mathrm{r}$ cells compared with that in U251s cells (Fig. 1). To explore the role of exosomal $\mathrm{Cx} 43$ in chemotherapy resistance, exosomes were initially isolated from U251s and U251r cells. Similar to parent cells, rExo expressed significantly higher $\mathrm{Cx} 43$ levels than sExo (Fig. 3A and C). Prior to incorporation, exosomes must dock and fuse with recipient cells. Thus, it is necessary to examine the role of exosomal $\mathrm{Cx} 43$ in exosome uptake by glioma cells. Although sExo and rExo uptake was observed in U251s cells, rExo with higher $\mathrm{Cx} 43$ expression was more readily incorporated into cells compared with sExo. In addition, the synthetic connexin 43 mimetic peptide ${ }^{37,43} \mathrm{Gap} 27$ possesses conserved sequence homology to a portion of the second extracellular loop leading into the fourth transmembrane connexin segment, and is widely used as a specific gap junction inhibitor directed against $\mathrm{Cx} 43(42,43)$. It has been reported that ${ }^{37,43} \mathrm{Gap} 27(100 \mathrm{nM}-100 \mu \mathrm{M})$ attenuated hemi-channel activity in adult keratinocytes and fibroblasts sourced from juvenile foreskin. The expression level of $\mathrm{Cx} 43$ was also decreased when JF and AK cells were exposed to ${ }^{37,43}$ Gap27 for $24 \mathrm{~h}$ (44). In the present study, ${ }^{37,43} \mathrm{Gap} 27$ directed against $\mathrm{Cx} 43$ markedly alleviated the uptake of rExo by U251s cells (Fig. 3E). These results suggest that exosomal $\mathrm{Cx} 43$ regulates the process of incorporation of exosomes into recipient cells. However, the mechanism underlying the role of exosomal $\mathrm{Cx} 43$ in exosome uptake remains to be further elucidated. It will be interesting to examine whether exosomal Cx43 cooperates with other membrane component molecules to facilitate docking or fusion with recipient cells. Next, the effect of exosomal $\mathrm{Cx} 43$ on the sensitivity of glioma cells to $\mathrm{TMZ}$ was examined. Treatment with rExo significantly increased the $\mathrm{IC}_{50}$ value of U251s cells to $\mathrm{TMZ}$ and colony formation, whereas ${ }^{37,43} \mathrm{Gap} 27$ efficiently inhibited the effect of rExo on U251s cells (Fig. 4A-D). TMZ induces DNA damage and cell cycle arrest, which leads to an imbalance of Bcl-2 and Bax and activates the intrinsic apoptosis pathway in glioma cells (45). It was previously demonstrated that $\mathrm{Cx} 43$ reduces the sensitivity of human LN18 and LN229 glioma cells to TMZ by reducing the $\mathrm{Bax} / \mathrm{Bcl}-2$ ratio and the release of cytochrome $c$ (29). Overexpression of $\mathrm{Cx} 43$ promotes the migration of U251 cells and inhibits apoptosis by upregulating Bcl-2 and downregulating the expression of Bax and caspase-3 (46). Thus, the effect of rExo on the expression of Bcl-2, Bax and cleaved caspase- 3 in U251s cells was examined. As expected, rExo increased the expression of Bcl-2 and decrease Bax and cleaved caspase-3 in U251s cells, while ${ }^{37,43}$ Gap27 alleviated the rExo-induced changes in the expression of Bax, Bcl-2 and cleaved caspase-3 in U251s cells (Fig. 5A-C). There are at least two plausible explanations for the role of exosomal Cx43 in the resistance of glioma cells to TMZ. First, the exosomal Cx43 may enhance TMZ resistance through modulating the expression of the pro-apoptotic and anti-apoptotic proteins and inhibiting the intrinsic apoptosis pathway $(29,46)$. Second, high expression of Cx43 in rExo may contribute to the formation of gap junction channels between exosomes and recipient cells, which facilitates transferring various TMZ resistance-related factors from exosomes to U251s cells. Furthermore, the role of exosomal Cx43 in the migration of glioma cells was also examined. The results of the wound healing and Transwell assays demonstrated that rExo promoted the migration of U251s cells, and ${ }^{37,43} \mathrm{Gap} 27$ efficiently alleviated rExo-induced 
cell migration (Fig. 6). In previous studies, gap junctions have been demonstrated to serve as an important regulator of cell migration during tumor metastasis, although some results are controversial. For example, increased expression of Cx26 has been reported to enhance the invasion of lung squamous cell carcinoma (47). However, overexpression of Cx26 and Cx43 has also been found to slow down the migration of breast cancer cells $(48,49)$. By contrast, the present study demonstrated that rExo with high $\mathrm{Cx} 43$ expression accelerated the migration of U251s cells. One possible explanation is that $\mathrm{Cx} 43$-assembled gap junction channels facilitate the delivery of effector molecules promoting migration from exosomes to $\mathrm{U} 251 \mathrm{~s}$ cells. Another explanation is that the response of glioma cells may differ from that of breast cancer cells.

In conclusion, the results of the present study may provide new insight into the role of exosomal $\mathrm{Cx} 43$ in the resistance of glioma cells to TMZ and cell migration. $\mathrm{Cx} 43$ was found to be highly expressed in TMZ-resistant U251r cells and rExo. Furthermore, treatment with rExo enhanced the resistance of U251s cells to TMZ, their colony formation and migration abilities, and changeed the expression pattern of Bax and Bcl-2. ${ }^{37,43}$ Gap27 directed against exosomal Cx43 significantly attenuated rExo-induced TMZ resistance, colony formation, cell migration and alterations in the expression of Bax and Bcl-2. These results indicate the important role of exosomal Cx43 in TMZ resistance and migration of glioma cells, and suggest a promising new strategy for preventing chemotherapy resistance and reducing the aggressiveness of glioblastoma by targeting exosomal $\mathrm{Cx} 43$ in the future.

\section{Acknowledgements}

Not applicable.

\section{Funding}

The present study was supported by the National Natural Science Foundation of China (grant nos. 81660014 and 81660159), the Key Program of the Natural Science Foundation of Jiangxi Province (grant no. 20202ACB206001), the Key Research and Development Program of Jiangxi Province (grant nos. 20192BBG70012 and 20192BBG70049), and the Research Fund for Key Laboratory of Drug Targets and Drug Screening of Jiangxi Province (grant no. 20171BCD40007).

\section{Availability of data and materials}

The datasets generated and analyzed during the present study are available from the corresponding author on reasonable request.

\section{Authors' contributions}

LPJ and XJH designed the current project. ZJY, LLZ, QCB, LJG, RJT, XML and LHL performed the experiments. LPJ, XJH, TH, MJW, ZJY and LLZ analyzed the data. ZJY, XJH and LLZ drafted the manuscript. LPJ, XJH and TH supervised the study. LPJ and XJH have seen and can confirm the authenticity of the raw data. All the authors have read and approved the final version of the manuscript.

\section{Ethics approval and consent to participate}

Not applicable.

\section{Patient consent for publication}

Not applicable.

\section{Competing interests}

The authors declare that they have no competing interests.

\section{References}

1. Lara-Velazquez M, Al-Kharboosh R, Jeanneret S, VazquezRamos C, Mahato D, Tavanaiepour D, Rahmathulla G and Quinones-Hinojosa A: Advances in brain tumor surgery for glioblastoma in adults. Brain Sci 20: 166, 2017.

2. Iwadate Y: Epithelial-mesenchymal transition in glioblastoma progression. Oncol Lett 11: 1615-1620, 2016.

3. Bonavia R, Inda MM, Cavenee WK and Furnari FB Heterogeneity maintenance in glioblastoma: A social network. Cancer Res 71: 4055-4060, 2011

4. Stupp R, Mason WP, van den Bent MJ, Weller M, Fisher B, Taphoorn MJ,Belanger K, Brandes AA, Marosi C, Bogdahn U, et al: Radiotherapy plus concomitant and adjuvant temozolomide for glioblastoma. New Engl J Med 352: 987-996, 2005.

5. Alonso MM, Gomez-Manzano C, Bekele BN, Yung WK and Fueyo J: Adenovirus-based strategies overcome temozolomide resistance by silencing the O6-methylguanine-DNA methyltransferase promoter. Cancer Res 67: 11499-11504, 2007.

6. Zhang J, Stevens MF and Bradshaw TD: Temozolomide: Mechanisms of action, repair and resistance. Curr Mol Pharmacol 5: 102-114, 2012.

7. Omar AI and Mason WP: Temozolomide: The evidence for its therapeutic efficacy in malignant astrocytomas. Core Evid 4: 93-111, 2010.

8. Neyns B, Tosoni A, Hwu WJ and Reardon DA: Dose-dense temozolomide regimens: Antitumor activity, toxicity, and immunomodulatory effects. Cancer 116: 2868-2877, 2010.

9. Garcia-Mayea Y, Mir C, Masson F, Paciucci R and ME LL: Insights into new mechanisms and models of cancer stem cell multidrug resistance. Semin Cancer Biol 60: 166-180, 2020.

10. Munoz JL, Walker ND, Mareedu S, Pamarthi SH, Sinha G, Greco SJ and Rameshwar P: Cycling quiescence in temozolomide resistant glioblastoma cells is partly explained by microRNA-93 and -193-mediated decrease of cyclin D. Front Pharmacol 10: $134,2019$.

11. Cao X, Lu Y, Liu Y, Zhou Y, Song H, Zhang W, Davis D, Cui J, Hao S, Jung J, et al: Combination of PARP inhibitor and temozolomide to suppress chordoma progression. J Mol Med (Berl) 97: 1183-1193, 2019.

12. Théry C, Zitvogel L and Amigorena S: Exosomes: Composition, biogenesis and function. Nat Rev Immunol 2: 569-579, 2002.

13. Ratajczak J, Wysoczynski M, Hayek F, Janowska-Wieczorek A and Ratajczak MZ: Membrane-derived microvesicles: Important and underappreciated mediators of cell-to-cell communication. Leukemia 20: 1487-1495, 2006.

14. Mathivanan S, Ji H and Simpson RJ: Exosomes: Extracellular organelles important in intercellular communication. J Proteomics 73: 1907-1920, 2010.

15. Cocucci E and Meldolesi J: Ectosomes and exosomes: Shedding the confusion between extracellular vesicles. Trends Cell Biol 25: 364-372, 2015.

16. Azmi AS, Bao B and Sarkar FH: Exosomes in cancer development, metastasis, and drug resistance: A comprehensive review. Cancer Metastasis Rev 32: 623-642, 2013.

17. Xiao GY, Cheng CC, Chiang YS, Cheng WT, Liu IH and Wu SC: Exosomal miR-10a derived from amniotic fluid stem cells preserves ovarian follicles after chemotherapy. Sci Rep 6: 23120, 2016.

18. Tanaka S, Hosokawa M, Ueda K and Iwakawa S: Effects of decitabine on invasion and exosomal expression of miR-200c and miR-141 in oxaliplatin-resistant colorectal cancer cells. Biol Pharm Bull 38: 1272-1279, 2015. 
19. O'Brien K, Lowry MC, Corcoran C, Martinez VG, Daly M, Rani S, Gallagher WM, Radomski MW, MacLeod RA and O'Driscoll L: MiR-134 in extracellular vesicles reduces triple-negative breast cancer aggression and increases drug sensitivity. Oncotarget 6: 32774-32789, 2015.

20. Santos JC, da Silva Lima N, Sarian LO, Matheu A, Ribeiro ML and Derchain SFM: Exosome-mediated breast cancer chemoresistance via miR-155 transfer. Sci Rep 8: 829, 2018.

21. Mulcahy LA, Pink RC and Carter DR: Routes and mechanisms of extracellular vesicle uptake. J Extracell Vesicles 3: 3402, 2014

22. Feng D, Zhao WL, Ye YY, Bai XC, Liu RQ, Chang LF, Zhou Q and Sui SF: Cellular internalization of exosomes occurs through phagocytosis. Traffic 11: 675-687, 2010.

23. Mincheva-NilssonLand Baranov V:Cancerexosomes and NKG2D receptor-ligand interactions: Impairing NKG2D-mediated cytotoxicity and anti-tumour immune surveillance. Semin Cancer Biol 28: 24-30, 2014.

24. Soares AR, Martins-Marques T, Ribeiro-Rodrigues T, Ferreira JV, Catarino S, Pinho MJ, Zuzarte M, Anjo SI, Manadas B, Sluijter JP, et al: Gap junctional protein $\mathrm{Cx} 43$ is involved in the communication between extracellular vesicles and mammalian cells. Sci Rep 5: 13243, 2015.

25. Scemes E, Spray DC and Meda P: Connexins, pannexins, innexins: Novel roles of 'hemi-channels'. Pflugers Arch 457: 1207-1226, 2009.

26. Maes M, Decrock E, Cogliati B, Oliveira AG, Marques PE, Dagli ML, Menezes GB, Mennecier G, Leybaert L, Vanhaecke T, et al: Connexin and pannexin (hemi)channels in the liver. Front Physiol 4: 405, 2014.

27. Aasen T, Mesnil M, Naus CC, Lampe PD and Laird DW: Gap junctions and cancer: Communicating for 50 years. Nat Rev Cancer 16: 775-788, 2016.

28. Sin WC, Crespin S and Mesnil M: Opposing roles of connexin43 in glioma progression. Biochim Biophys Acta 1818: 2058-2067, 2012.

29. Gielen PR, Aftab Q, Ma N, Chen VC, Hong X, Lozinsky S, Naus CC and Sin WC: Connexin43 confers temozolomide resistance in human glioma cells by modulating the mitochondrial apoptosis pathway. Neuropharmacology 75: 539-548, 2013.

30. Theis $\mathrm{M}$ and Giaume C: Connexin-based intercellular communication and astrocyte heterogeneity. Brain Res 1487: 88-98, 2012.

31. Caltabiano R, Torrisi A, Condorelli D, Albanese V and Lanzafame S: High levels of connexin 43 mRNA in high grade astrocytomas. Study of 32 cases with in situ hybridization. Acta Histochem 112: 529-535, 2010.

32. Munoz JL, Rodriguez-Cruz V, Greco SJ, Ramkissoon SH, Ligon KL and Rameshwar P: Temozolomide resistance in glioblastoma cells occurs partly through epidermal growth factor receptor-mediated induction of connexin 43. Cell Death Dis 5: e1145, 2014.

33. Helwa I, Cai J, Drewry MD, Zimmerman A, Dinkins MB, Khaled ML, Seremwe M, Dismuke WM, Bieberich E, Stamer WD, et al: A comparative study of serum exosome isolation using differential ultracentrifugation and three commercial reagents. PLoS One 12: e0170628, 2017.

34. Han XJ, Yang ZJ, Jiang LP, Wei YF, Liao MF, Qian Y, Li Y, Huang X, Wang JB, Xin HB and Wan YY: Mitochondrial dynamics regulates hypoxia-induced migration and antineoplastic activity of cisplatin in breast cancer cells. Int J Oncol 46: 691-700, 2015.

35. Konadu KA, Huang MB, Roth W, Armstrong W, Powell M, Villinger $\mathrm{F}$ and Bond $\mathrm{V}$ : Isolation of exosomes from the plasma of HIV-1 positive individuals. J Vis Exp 5: 53495, 2016.
36. Das A, Banik NL, Patel SJ and Ray SK: Dexamethasone protected human glioblastoma U87MG cells from temozolomide induced apoptosis by maintaining Bax:Bcl-2 ratio and preventing proteolytic activities. Mol Cancer 3: 36, 2004.

37. Fan QW, Cheng C, Hackett C, Feldman M, Houseman BT, Nicolaides T, Haas-Kogan D, James CD, Oakes SA, Debnath J, et al: Akt and autophagy cooperate to promote survival of drug-resistant glioma. Sci Signal 3: ra81, 2010.

38. Chistiakov DA and Chekhonin VP: Extracellular vesicles shed by glioma cells: Pathogenic role and clinical value. Tumour Biol 35: 8425-8438, 2014.

39. Jia G, Han Y, An Y, Ding Y, He C, Wang X and Tang Q: NRP-1 targeted and cargo-loaded exosomes facilitate simultaneous imaging and therapy of glioma in vitro and in vivo. Biomaterials 178 : 302-316, 2018.

40. Goodenough DA and Paul DL: Beyond the gap: Functions of unpaired connexon channels. Nat Rev Mol Cell Biol 4: 285-294, 2003.

41. Murphy SF, Varghese RT, Lamouille S, Guo S, Pridham KJ, Kanabur P, Osimani AM, Sharma S, Jourdan J, Rodgers CM, et al: Connexin 43 inhibition sensitizes chemoresistant glioblastoma cells to temozolomide. Cancer Res 76: 139-149, 2016.

42. Ilvesaro J, Tavi P and Tuukkanen J: Connexin-mimetic peptide gap 27 decreases osteoclastic activity. BMC Musculoskelet Disord 2: 10, 2001.

43. Edwards G, Félétou M, Gardener MJ, Thollon C, Vanhoutte PM and Weston AH: Role of gap junctions in the responses to EDHF in rat and guinea-pig small arteries. Br J Pharmacol 128: 1788-1794, 1999.

44. Faniku C, O'Shaughnessy E, Lorraine C, Johnstone SR, Graham A, Greenhough S and Martin PE: The connexin mimetic peptide gap27 and $\mathrm{Cx} 43$-knockdown reveal differential roles for connexin43 in wound closure events in skin model systems. Int J Mol Sci 19: 604, 2018.

45. Hombach-Klonisch S, Mehrpour M, Shojaei S, Harlos C, Pitz M, Hamai A, Siemianowicz K, Likus W, Wiechec E, Toyota BD, et al: Glioblastoma and chemoresistance to alkylating agents: Involvement of apoptosis, autophagy, and unfolded protein response. Pharmacol Ther 184: 13-41, 2018.

46. Lu J, Yu M, Lin Z, Lue S, Zhang H, Zhao H, Xu Y and Liu H: Effects of connexin43 overexpression on U251 cell growth, migration, and apoptosis. Med Sci Monit 23: 2917-2923, 2017.

47. Ito A, Koma Y, Uchino K, Okada T, Ohbayashi C, Tsubota N and Okada M: Increased expression of connexin 26 in the invasive component of lung squamous cell carcinoma: Significant correlation with poor prognosis. Cancer Lett 234: 239-248, 2006.

48. Kalra J, Shao Q, Qin H, Thomas T, Alaoui-Jamali MA and Laird DW: Cx26 inhibits breast MDA-MB-435 cell tumorigenic properties by a gap junctional intercellular communicationindependent mechanism. Carcinogenesis 27: 2528-2537, 2006.

49. McLachlan E, Shao Q, Wang HL, Langlois S and Laird DW: Connexins act as tumor suppressors in three-dimensional mammary cell organoids by regulating differentiation and angiogenesis. Cancer Res 66: 9886-9894, 2006.

This work is licensed under a Creative Commons Attribution-NonCommercial-NoDerivatives 4.0 International (CC BY-NC-ND 4.0) License. 